\title{
Increased oxidative stress and disrupted small intestinal tight junctions in cigarette smoke-exposed rats
}

\author{
HONGWEI LI ${ }^{1 *}$, QI WU ${ }^{2 *}$, LONG XU ${ }^{2}$, XUE LI $^{2}$, JIANMIN DUAN $^{1}$, JINGYAN ZHAN $^{1}$, \\ JING FENG ${ }^{3}, \mathrm{XIN} \mathrm{SUN}^{4}$ and HUAIYONG CHEN ${ }^{2}$ \\ ${ }^{1}$ Graduate School, Tianjin Medical University, Tianjin 300070; ${ }^{2}$ Department of Basic Medicine Laboratory, \\ Tianjin Institute of Respiratory Diseases, Haihe Hospital, Tianjin 300350; ${ }^{3}$ Respiratory Department, \\ Tianjin Medical University General Hospital, Tianjin 300052; ${ }^{4}$ Key Research Laboratory for Infectious \\ Disease Prevention for State Administration of Traditional Chinese Medicine, Respiratory Department, \\ Tianjin Institute of Respiratory Diseases, Haihe Hospital, Tianjin 300350, P.R. China
}

Received February 25, 2014; Accepted January 7, 2015

DOI: $10.3892 / \mathrm{mmr} .2015 .3234$

\begin{abstract}
Chronic obstructive pulmonary disease (COPD) is a major public health problem, and cigarette smoke (CS) is the primary risk factor. The pathology is often observed in the lung, but COPD is also associated with intestinal barrier disruption, although the underlying mechanisms are poorly understood. To address this, a CS-exposed rat model was evaluated in the present study by analyzing small intestinal gene expression using reverse transcription-quantitative polymerase chain reaction. CS exposure caused upregulation of the nicotinamide adenine dinucleotide phosphate-oxidase subunits nox 2 and p2 $2^{\text {phox }}$ in the small intestine, while the antioxidative enzyme superoxide dismutase was downregulated. CS exposure also increased bax expression and decreased bcl-2 expression. This was associated with an elevation of hypoxia-inducible factor (HIF)-1 $\alpha$. Claudin-1 was decreased and claudin-2 increased, indicating a loosening of small intestinal tight junctions (TJs). These data suggest that during the development of COPD, HIF-1 $\alpha$ expression is altered in the small intestine, which may be associated with the increased oxidative stress and apoptosis, eventually resulting in disruption of the intestinal TJs.
\end{abstract}

Correspondence to: Dr Xin Sun, Key Research Laboratory for Infectious Disease Prevention for State Administration of Traditional Chinese Medicine, Respiratory Department, Tianjin Institute of Respiratory Diseases, Haihe Hospital, 50 Jingu Road, Tianjin 300350, P.R. China

E-mail: 13302031999@126.com

Dr Huaiyong Chen, Department of Basic Medicine Laboratory, Tianjin Institute of Respiratory Diseases, Haihe Hospital, 50 Jingu Road, Tianjin 300350, P.R. China

E-mail: huaiyong.chen@foxmail.com

*Contributed equally

Key words: cigarette smoke, small intestine, tight junction, claudin-1, claudin-2, hypoxia-inducible factor $1 \alpha$

\section{Introduction}

Chronic obstructive pulmonary disease (COPD) is characterized by persistent airflow limitation, and is a major public health problem (1). By the year 2030, COPD is predicted to have become the seventh most common disease and the fourth leading cause of mortality worldwide (2). Cigarette smoking is the most common risk factor for developing COPD, and the disease prevalence in numerous countries is associated with the rate of smoking (3). COPD also has a proven association with persistent systemic inflammation, excessive oxidative stress and abnormal immune function (4). These numerous effects highlight its multidimensional nature; although COPD damage is primarily observed in the lungs, it is often accompanied by disruptions to cardiovascular, musculoskeletal, renal and intestinal function (5).

Small intestinal integrity is maintained by intercellular tight junctions (TJs) between luminal cells. TJs regulate solute and ion transport through the paracellular pathway (6), which is the dominant pathway for passive transepithelial solute flow in the small intestine (7). A number of compounds regulate TJs, including transmembrane proteins such as occludin and claudins, and peripheral membrane proteins such as zonula occludens (ZOs). Claudins are integral TJ proteins regulating the size selectivity of the barrier, occludin is considered to provide the primary intercellular seal between epithelial cells, and ZOs provide the critical connection between transmembrane TJ components and the intracellular actin cytoskeleton (8).

TJ protein expression is regulated by extracellular stimuli including oxidative stress responses and pro-inflammatory cytokines $(9,10)$. Hypoxia-inducible factor 1 (HIF-1), a transcription factor regulating oxidative stress and apoptotic genes (11), is a notable modulator of TJ protein expression $(12,13)$. HIF-1 is selectively stabilized and activated during local hypoxia, and coordinates the tissue's adaptive response to hypoxia by facilitating oxygen supply, glucose transport and angiogenesis (14). Functionally, HIF-1 is a heterodimer comprising an inducible HIF-1 $\alpha$ subunit and a constitutively expressed HIF-1 $\beta$ subunit. HIF-1 $\alpha$ is upregulated in response to hypoxia and accounts for the disruption of the intestinal luminal structure (15). 
Table I. DNA primer sequences for quantitative reverse transcription-quantitative polymerase chain reaction.

\begin{tabular}{lll}
\hline Gene & \multicolumn{1}{c}{ Forward primer } & \multicolumn{1}{c}{ Reverse primer } \\
\hline GAPDH & 5'-TGGAGTCTACTGGCGTCTTC-3' & 5'-TTCACACCCATCACAAACATG-3' \\
claudin-1 & 5'-TGTCCACCATTGGCATGAAG-3' & 5'-GCCACTAATGTCGCCAGACC-3' \\
claudin-2 & 5'-ACAGCACTGGCATCACCCA-3' & 5'-GCGAGGACATTGCACTGGAT-3' \\
claudin-4 & 5'-AAGGCCAAGGTCATGATCACAG-3' & 5'-GAAGTCGCGGATGACGTTGT-3' \\
occludin & 5'-CTACTCCTCCAACGGCAAAG-3' & 5'-AGTCATCCACGGACAAGGTC-3' \\
zo-1 & 5'-ATTCAGTTCGCTCCCATGAC-3' & 5'-GCTGTGGAGACTGTGTGGAA-3' \\
HIF-1 $\alpha$ & 5'-AAGAAACCGCCTATGACGTG-3' & 5'-CCACCTCTTTTTGCAAGCAT-3' \\
bax & 5'-CCAGGACGCATCCACCAAGAAGC-3' & 5'-TGCCACACGGAAGAAGACCTCTCG-3' \\
bcl-2 & 5'-GGATGACTTCTCTCGTCGCTACCGT-3' & 5'-CGAGTGAGGATGTGCATGAA-3' \\
SOD & 5'-GCAGAAGGCAAGCGGTGAAC-3' & 5'-TCACACCACAAGCCAAGCGG-3' \\
p22 ${ }^{\text {phox }}$ & 5'-AAGTACCTGACCGCTGTGG-3' & 5'-AGGTAGATCACACTGGCAATG-3' \\
nox2 & 5'-GGCTGTGAATGAGGGACTC-3' & 5'-CCAGTGCTGACCCAAGAAG-3' \\
TNF- $\alpha$ & 5'-CGTCGTAGCAAACCACCAAG-3' & 5'-CACAGAGCAATGACTCCAAAG-3' \\
NF- $k B$ & 5'-AGCCCTATGCCTTTTCAACAT-3' & 5'-CACTCCTGGGTCTGTGTTGTT-3'
\end{tabular}

GAPDH, glyceraldehyde 3-phosphate dehydrogenase; zo-1, zonula occludens 1; HIF-1 $\alpha$, hypoxia-inducible factor 1 $\alpha$; SOD, superoxide dismutase; TNF- $\alpha$, tumor necrosis factor $\alpha$.

Significantly, the respiratory and intestinal tracts have a similar luminal structure; the two organs have an extensive luminal surface area, selective epithelial barrier and overlying mucus-gel layer (16). The absorptive and barrier function of the intestinal epithelium is sensitive to tissue hypoxia and reduced perfusion, and similarly, tissue hypoxia is one of the most notable characteristics in COPD pathology (17). Clinical findings indicate a combination of disturbed intestinal integrity, intestinal hyperpermeability and enhanced enterocyte loss in COPD patients $(18,19)$. However, the underlying molecular mechanisms of this small intestinal damage remain unknown.

In the present study, we exposed rats to cigarette smoke (CS) to induce COPD, which is a well-established model (20). Gene expression analysis of intestinal samples by reverse transcription-quantitative polymerase chain reaction (RT-qPCR) was used to evaluate the effect of CS on oxidative stress and inflammation. The present study may provide an essential molecular mechanism for intestinal barrier dysfunction and hyperpermeability in patients with COPD.

\section{Materials and methods}

Ethics. Rats were used in strict accordance with the protocol approved by the Animal Care Committee of Tianjin Medical University, China.

Exposure of rats to cigarette smoke. Male Wistar rats weighing $180 \pm 20 \mathrm{~g}$ and aged 6 weeks were purchased from the Model Animal Center of the Radiological Medicine Research Institute, Chinese Academy of Medical Science (Tianjin, China). Rats were housed in standard laboratory cages (five per cage) and had free access to food and water. Rats were randomly divided into two groups ( $\mathrm{n}=15$ per group) matched by body weight; a CS-exposed group and an unexposed control group. As described previously (21), the CS group received whole-body exposure to the smoke of five unfiltered cigarettes (Daqianmen $^{\mathrm{TM}}$, tar $\leq 15 \mathrm{mg}$, nicotine $\leq 1.1 \mathrm{mg}$ and $\mathrm{CO} \leq 13 \mathrm{mg}$ ) for $30 \mathrm{~min}$ twice daily (before 9 am and after $5 \mathrm{pm}$ ), every day for 14 weeks inside a $0.6 \mathrm{~m}^{3}$ custom plexiglass chamber (made in-house), which included five ventilation holes. Naïve animals underwent an identical protocol but without CS exposure. Smoke contamination between the study groups was prevented by using different chambers for each.

Total RNA isolation. Following treatment, rats were anesthetized and sacrificed. The abdominal cavity was opened, and the duodenum was excised, rinsed in ice-cold phosphate-buffered saline ( $\mathrm{pH} 7.4$; Sangon Biotech, Shanghai, China), frozen in liquid nitrogen and stored at $-80^{\circ} \mathrm{C}$ until analysis. RNA was extracted from duodenum tissues using TRIzol reagent (Invitrogen, Carlsbad, CA, USA). Extract yield and quality were determined by measuring absorbance at 260 and $280 \mathrm{~nm}$ with the MaestroNano Micro-Volume spectrophotometer (Maestrogen, Inc., Las Vegas, NV, USA). The absorbance ratio at 260:280 was between 1.8 and 2.0. The RNA was subsequently reverse transcribed into complementary DNA (cDNA).

$R T$ - $q P C R$. Messenger RNA (mRNA; $3 \mu \mathrm{g}$ ) was reverse transcribed with oligo (dT) primer for $1 \mathrm{~h}$ at $50^{\circ} \mathrm{C}$ using a TIANScript RT kit (Tiangen Biotech Co., Ltd, Beijing, China) according to the manufacturer's instructions. The cDNA served as templates for RT-qPCR, which was performed using SYBR-Green PCR core reagents (Bio-Rad Laboratories, Hercules, CA, USA). Specific gene primers were designed using Primer-Quest SM software available at http://www. idtdna.com/Scitools/Applications/PrimerQuest/ (Integrated DNA Technologies, Inc., Coralville, IA, USA), then commercially produced (BGI Tech, Shenzhen, Guangdong, China; 

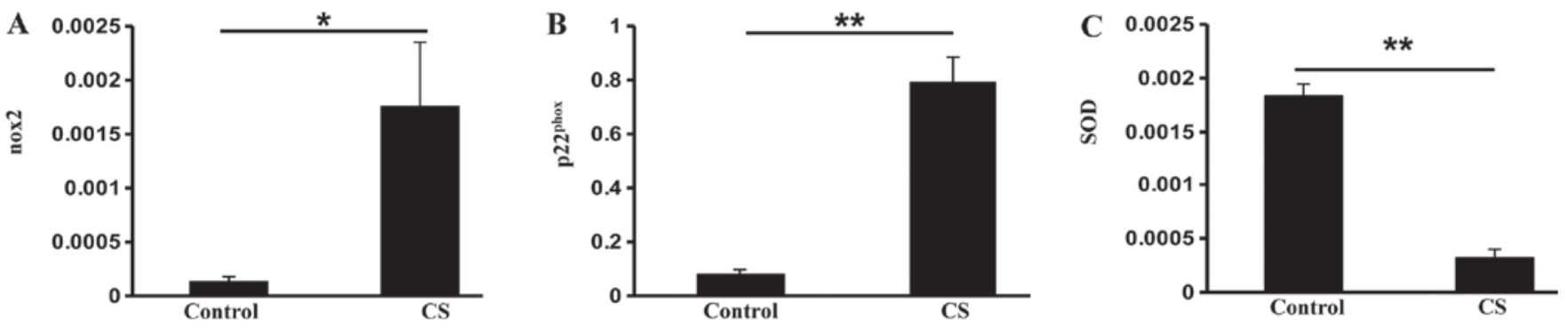

Figure 1. Oxidative stress-related gene expression in rat small intestine following cigarette smoke (CS) exposure. The expression of nox2 (A), p22 phox $(\mathrm{B})$ and superoxide dismutase (SOD) (C) was determined using reverse transcription-quantitative polymerase chain reaction on the small intestine of naïve rats ( $=3$ ) and CS-exposed rats $(n=3)$. The mRNA levels were normalized to glyceraldehyde 3-phosphate dehydrogenase levels. Data are expressed as the means \pm SEM. ${ }^{*} \mathrm{P}<0.05$ and ${ }^{* *} \mathrm{P}<0.01$ compared with naïve rats.
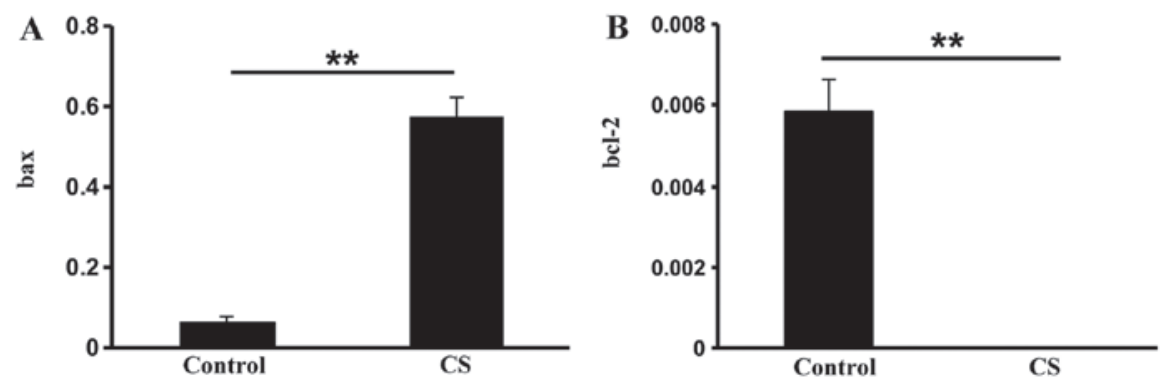

Figure 2. Apoptosis-related gene expression in rat small intestine following cigarette smoke (CS) exposure. Bax (A) and bcl-2 (B) mRNA expression was analyzed using reverse transcription-quantitative polymerase chain reaction. The mRNA levels were normalized to glyceraldehyde 3 -phosphate dehydrogenase levels. Data are expressed as the means \pm SEM. ${ }^{* *} \mathrm{P}<0.01$ compared with naïve rats.

Table I). DNA amplifications were performed on a CFX96 real-time system (Bio-Rad Laboratories) under the following reaction conditions: an initial heating cycle of $95^{\circ} \mathrm{C}$ for $2 \mathrm{~min}$; 40 cycles alternating between denaturation at $95^{\circ} \mathrm{C}$ for $25 \mathrm{sec}$ and primer annealing at $60^{\circ} \mathrm{C}$ for $25 \mathrm{sec}$; and extension at $72^{\circ} \mathrm{C}$ for $20 \mathrm{sec}$. Melt curves clarified the identity of amplicons, and the housekeeping gene, glyceraldehyde 3-phosphate dehydrogenase (GAPDH), served as an internal control. The relative mRNA expression of targeted genes was calculated by the comparative threshold cycle (CT) method normalized to GAPDH mRNA in the same sample. Briefly, specific $\Delta \mathrm{Ct}$ was calculated as follows: $\Delta \mathrm{Ct}=\left(\mathrm{Ct}_{\text {target }}\right)-\left(\mathrm{Ct}_{\mathrm{GAPDH}}\right)$; relative expression was defined as: $2^{-\Delta \mathrm{Ct}}$.

Statistical analysis. Results are presented as the means \pm SEM. Statistical comparisons were made using the one-tailed Student's t-test. $\mathrm{P}<0.05$ was considered to indicate a statistically significant difference.

\section{Results}

Exposure to CS affects oxidative stress-related genes. Both patients with COPD and animals exposed to CS demonstrate oxidative stress and inflammation systemically $(4,22)$. In this study, we exposed rats to CS and then evaluated the effects of CS exposure on the expression of genes that are associated with oxidative stress and inflammation in the small intestine of rats by RT-qPCR. There was a significant increase in the expression of the nicotinamide adenine dinucleotide phosphate (NADPH) oxidase subunits nox $2(\mathrm{P}<0.05)$ and $\mathrm{p} 22^{\text {phox }}(\mathrm{P}<0.01)$ in the $\mathrm{CS}$ group (Fig. 1A and B). However, the antioxidant enzyme superoxide dismutase (SOD) was reduced in the CS group compared with the control group $(\mathrm{P}<0.01$; Fig. 1C), indicating that $\mathrm{CS}$ exposure increases oxidative stress in the small intestine.

To investigate the correlation between CS-associated oxidative stress and intestinal apoptosis, we then measured pro-apoptotic bax and anti-apoptotic bcl-2 gene expression. The bax mRNA expression was over five times greater in the CS group compared with the control group ( $\mathrm{P}<0.01$; Fig. $2 \mathrm{~A}$ ). In addition, bcl-2 expression was significantly reduced in the CS group ( $\mathrm{P}<0.01$; Fig. 2B). These data suggested that CS exposure induces apoptosis in intestinal cells.

The transcription factor $\mathrm{NF}-\kappa \mathrm{B}$ was evaluated to assess small intestinal inflammation in the study groups. NF- $\kappa \mathrm{B}$ expression was higher in the CS group compared with the control group, but this change was not significant $(\mathrm{P}=0.09$; Fig. 3A). There was no significant difference in the expression of inflammatory cytokine tumor necrosis factor $\alpha$ (TNF- $\alpha$ ), which is controlled by NF- $\kappa \mathrm{B}$ (23), between the two groups (Fig. 3B). These data together suggested that CS exposure induces oxidative stress and apoptosis in the small intestine.

Exposure to CS upregulates HIF-1 $\alpha$. HIF-1 $\alpha$ is usually elevated during hypoxic stress, but it may also be induced by oxidative stress (24). The HIF-1 $\alpha$ subunit is selectively stabilized and primarily determines HIF-1 activity (14). Compared with the control rats, HIF-1 $\alpha$ expression was significantly increased in the CS rats $(\mathrm{P}<0.05$; Fig. 4). These data suggested that $\mathrm{CS}$ exposure may induce oxidative stress by upregulating HIF-1 in the small intestine. 

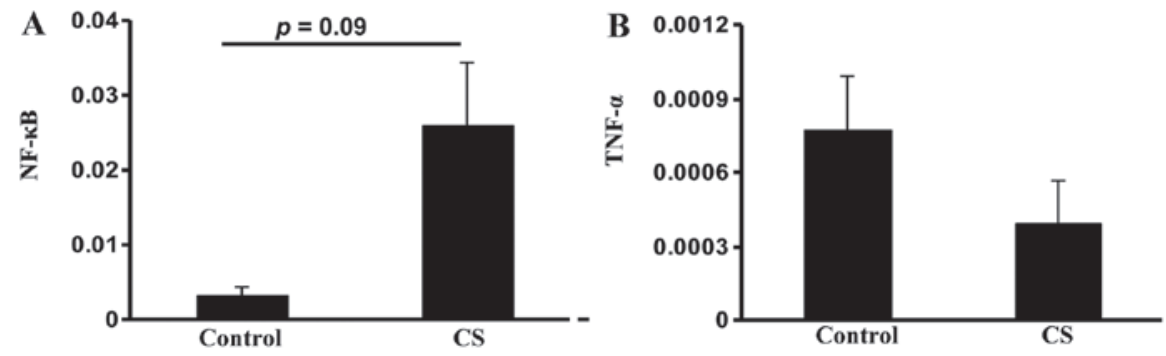

Figure 3. Inflammatory cytokine tumor necrosis factor (TNF)- $\alpha$ and nuclear transcription factor NF- $\mathrm{BB}$ expression in rat small intestine following cigarette smoke (CS) exposure. The mRNA expression of NF- $\mathrm{kB}(\mathrm{A})$ and TNF- $\alpha(B)$ in naïve $(n=3)$ and CS-exposed rats $(n=3)$ was determined using reverse transcription-quantitative polymerase chain reaction; no association between CS exposure and cytokine expression was observed. The mRNA levels were normalized to glyceraldehyde 3-phosphate dehydrogenase levels. Data are expressed as the means \pm SEM.

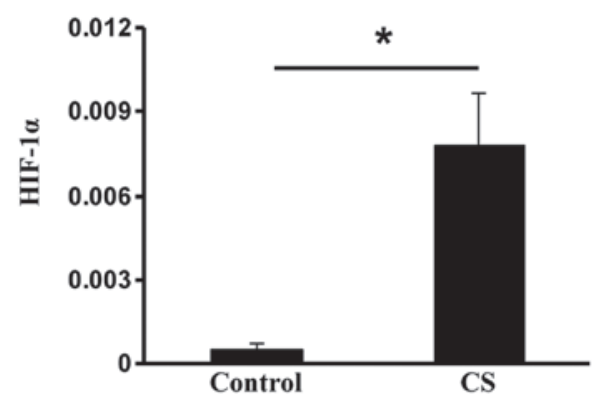

Figure 4. Small intestinal hypoxia-inducible factor (HIF)-1 $\alpha$ expression in rats following cigarette smoke (CS) exposure. HIF-1 $\alpha$ mRA expression, analyzed using reverse transcription-quantitative polymerase chain reaction, increased following CS exposure. The mRNA level was normalized to the glyceraldehyde 3-phosphate dehydrogenase level. Data are expressed as the means \pm SEM. ${ }^{*} \mathrm{P}<0.05$ compared with naïve rats.
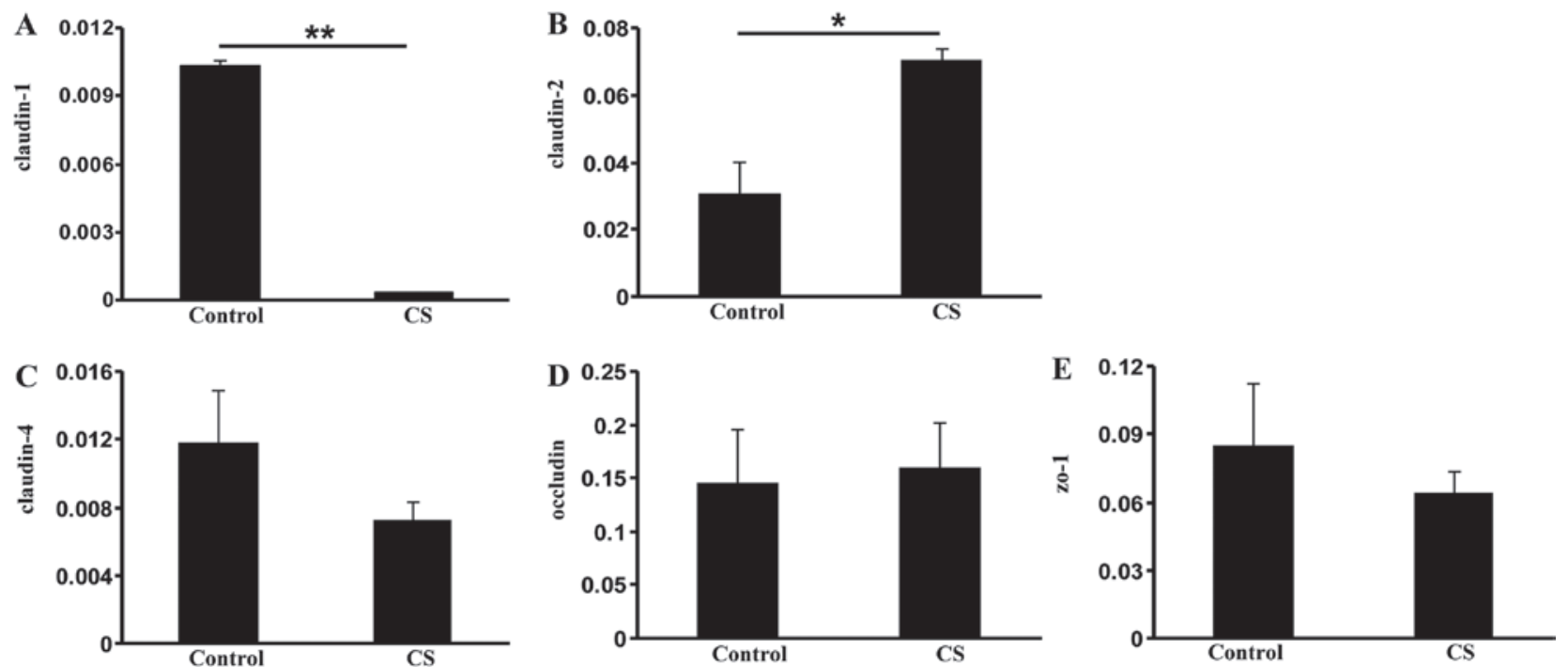

Figure 5. Tight junction protein mRNA expression in rat small intestine following cigarette smoke (CS) exposure. The small intestinal mRNA expression of claudin-1 (A), claudin-2 (B), claudin-4 (C), occludin (D) and zonula occludens (zo)-1 (E) in naïve rats (n=3) and CS-exposed rats ( $\mathrm{n}=3$ ) was determined using reverse transcription-quantitative polymerase chain reaction. The mRNA levels were normalized to glyceraldehyde 3-phosphate dehydrogenase levels. Data are expressed as the means \pm SEM. ${ }^{*} \mathrm{P}<0.05$ and ${ }^{* *} \mathrm{P}<0.01$ compared with naïve rats.

Exposure to CS alters expression of TJ protein genes. The activation of HIF-1 is associated with the disruption of TJs (25). To gain insight into the mechanism of increased intestinal permeability in COPD, we measured the expression of several TJ components: claudin-1, claudin-2, claudin-4, occludin and zo-1. Compared with the control group, the CS group demonstrated significantly reduced claudin-1 expression
$(\mathrm{P}<0.01$; Fig. 5A), indicating that $\mathrm{CS}$ exposure loosens the $\mathrm{TJ}$ of intestinal luminal cells (26). By contrast, elevated expression of claudin-2 $(\mathrm{P}<0.05)$, a leaky protein playing an opposing role to claudin-1 (27), was observed in the CS group compared with the control group (Fig. 5B). However, no significant changes in claudin-4, occludin and zo-1 expression were observed (Fig. 5C-E). These data suggest that CS exposure 
increases intestinal permeability through the dysregulation of TJ components.

\section{Discussion}

Patients with COPD exhibit disrupted intestinal integrity. To highlight the mechanisms underlying this disorder, we used a CS model, which revealed that CS exposure increases oxidative stress and induces apoptosis in the rat small intestine, while the inflammatory cytokine $\mathrm{TNF}-\alpha$ levels remained unchanged. Our data suggested that the disrupted intestinal barrier in COPD may be associated with HIF-1 $\alpha$ elevation, which causes dysregulation of TJ components.

Tissue hypoxia is a key player in a number of the extrapulmonary comorbidities occurring in COPD (17). This hypoxia is primarily driven by a ventilation/perfusion mismatch resulting from progressive airflow limitation and emphysematous destruction of the pulmonary capillary bed. The risk of alveolar hypoxia and subsequent hypoxemia increases as the disease progresses (1). Hypoxia caused by COPD greatly impacts the expression of oxygen-dependent genes, including HIF-1 $\alpha$. Aside from oxygen-dependent regulation, HIF-1 $\alpha$ expression is also regulated by growth factors and free radicals. Studies reveal that nicotine exposure, a significant component of cigarettes, mimics hypoxic effects by increasing the generation of mitochondrial reactive oxidative species (ROS) and thus stimulates HIF-1 $\alpha$ accumulation and activity (28). An oxidative stress response frequently follows smoke exposure, and potentially, hypoxic stimulation in the CS exposure model worsened the oxidative stress response in the small intestine, resulting in HIF-1 $\alpha$ upregulation.

The roles of HIF-1 $\alpha$ in the regulation of barrier function remain controversial. One study suggests that HIF- $1 \alpha$ is a protective factor for intestinal barrier function, as demonstrated in experimental murine colitis (29). However, another study reveals that HIF-1 $\alpha$ injures the barrier function and TJs (12). Prolonged intestinal HIF-1 $\alpha$ exposure is associated with potential ischemia/reperfusion-induced gut mucosal injury, which contributes to gut barrier function loss, bacterial translocation, apoptosis, gut-derived inflammatory response and resulting villous injury (30). The adverse effects of HIF-1 $\alpha$ are apparently mediated by intestinal bacteria or bacterial products (31). Hence, HIF-1 $\alpha$ appears to be involved intestinal barrier regulation, but its role, whether protective or injurious, may depend on the exact physiological conditions. Reportedly, HIF-1 $\alpha$ upregulation is coupled to a reduction in zo-1 protein concentration and dislocation of zo-1 from the TJ, which enables redistribution of other TJ proteins, including claudin-1 (13). HIF-1 $\alpha$ suppression is correlated with attenuated intestinal barrier dysfunction and morphologic redistribution of TJ proteins zo-1, occludin and claudin-1, triggered by pro-inflammatory cytokines (32). Therefore, HIF-1 $\alpha$ may cause small intestinal epithelial barrier damage and hyperpermeability through TJ protein regulation.

Only claudin-1 and claudin-2 were affected by CS exposure in the present study, suggesting that CS selectively affects different TJ components. This selective effect has been previously observed (33). In a side-stream smoking study, increased $\mathrm{TJ}$ protein expression and reduced inflammation were observed in the large intestine (34). The divergence between these studies may result from the different smoke simulation methods and variable effect on the different intestinal segments evaluated by the studies.

Increased small intestine apoptosis following CS exposure has also been observed by other groups (35). HIF-1 $\alpha$ induces apoptosis through an overexpression of pro-apoptotic proteins at the transcriptional level (36), which bind and inhibit the anti-apoptotic protein bcl-2 (37). Bcl-2 expression is correlated with intestinal damage and TJ breakdown (38). However, it is unclear whether intestinal cell apoptosis is the primary event associated with CS or whether it is induced indirectly by HIF-1 $\alpha$. Cell apoptosis can be induced by oxidative stress, which may impair intestinal epithelial integrity and TJs. ROS-induced damage represents an essential mechanism contributing to the TJ barrier defect (9). The antioxidant enzyme SOD is the first line of tissue defense against ROS. SOD expression increased in the rat small intestine following CS exposure in the present study, in line with a previous study (39). This may indicate a decreased radical-scavenging ability of the intestine following smoke exposure, increasing gut susceptibility to oxidative damage. In addition, the NADPH oxidase subunits, nox 2 and p22 $2^{\text {phox }}$, are elevated in the CS intestine; NADPH oxidase is potentially a source of ROS in CS exposure. Notably, COPD development is associated with NADPH oxidase increase (40), and this same redox status may occur in the small intestine in patients with COPD. Future analysis of intestinal protein expression using western blotting or immunohistochemistry is required to confirm this hypothesis.

In conclusion, $\mathrm{CS}$ exposure elevates oxidative stress, apoptosis and TJ component dysregulation, potentially mediated by HIF-1 $\alpha$. These data provide new insight into the mechanisms behind cigarette smoke and associated intestinal mucosal disruption in COPD patients.

\section{Acknowledgements}

This study was supported by the Natural Science Foundation of Tianjin City (13JCYBJC22400 and 13JCYBJC40000) and the National Natural Science Foundation of China (81270144).

\section{References}

1. Vestbo J, Hurd SS, Agusti AG, et al: Global strategy for the diagnosis, management, and prevention of chronic obstructive pulmonary disease: GOLD executive summary. Am J Respir Crit Care Med 187: 347-365, 2013.

2. Mathers CD and Loncar D: Projections of global mortality and burden of disease from 2002 to 2030. PLoS Med 3: e442, 2006.

3. Pauwels RA and Rabe KF: Burden and clinical features of chronic obstructive pulmonary disease (COPD). Lancet 364: 613-620, 2004.

4. Celli BR: Update on the management of COPD. Chest 133: 1451-1462, 2008.

5. Divo M, Cote C, de Torres JP, et al: Comorbidities and risk of mortality in patients with chronic obstructive pulmonary disease. Am J Respir Crit Care Med 186: 155-161, 2012.

6. Tsukita S, Furuse M and Itoh M: Multifunctional strands in tight junctions. Nat Rev Mol Cell Biol 2: 285-293, 2001.

7. Anderson JM and Van Itallie CM: Tight junctions and the molecular basis for regulation of paracellular permeability. Am J Physiol 269: G467-G475, 1995.

8. Mitic LL, Van Itallie CM and Anderson JM: Molecular physiology and pathophysiology of tight junctions I. Tight junction structure and function: lessons from mutant animals and proteins. Am J Physiol Gastrointest Liver Physiol 279: G250-G254, 2000. 
9. Zhu $\mathrm{H}$ and Li YR: Oxidative stress and redox signaling mechanisms of inflammatory bowel disease: updated experimental and clinical evidence. Exp Biol Med (Maywood) 237: 474-480, 2012.

10. Bruewer M, Luegering A, Kucharzik T, et al: Proinflammatory cytokines disrupt epithelial barrier function by apoptosisindependent mechanisms. J Immunol 171: 6164-6172, 2003.

11. Lee KA, Roth RA and LaPres JJ: Hypoxia, drug therapy and toxicity. Pharmacol Ther 113: 229-246, 2007.

12. Rosenberger P, Khoury J, Kong T, Weissmuller T, Robinson AM and Colgan SP: Identification of vasodilator-stimulated phosphoprotein (VASP) as an HIF-regulated tissue permeability factor during hypoxia. FASEB J 21: 2613-2621, 2007.

13. Nico B, Mangieri D, Crivellato E, et al: HIF activation and VEGF overexpression are coupled with ZO-1 up-phosphorylation in the brain of dystrophic mdx mouse. Brain Pathol 17: 399-406, 2007.

14. Lee JW, Bae SH, Jeong JW, Kim SH and Kim KW: Hypoxia-inducible factor (HIF-1)alpha: its protein stability and biological functions. Exp Mol Med 36: 1-12, 2004

15. Semenza GL: Regulation of oxygen homeostasis by hypoxia-inducible factor 1. Physiology (Bethesda) 24: 97-106, 2009.

16. Keely S, Talley NJ and Hansbro PM: Pulmonary-intestinal cross-talk in mucosal inflammatory disease. Mucosal Immunol 5: 7-18, 2012

17. Kent BD, Mitchell PD and McNicholas WT: Hypoxemia in patients with COPD: cause, effects, and disease progression. Int J Chron Obstruct Pulmon Dis 6: 199-208, 2011.

18. Rutten EP, Lenaerts K, Buurman WA and Wouters EF: Disturbed intestinal integrity in patients with COPD: effects of activities of daily living. Chest 145: 245-252, 2014.

19. Beloborodova EI, Akimova LA, Kritskaia NG, Asanova AV, Semenenko EV and Burkovskaia VA: Disturbed absorptive function of small intestines in patients with chronic obstructive pulmonary disease. Klin Med (Mosk) 90: 54-59, 2012 (In Russian)

20. Wright JL, Cosio M and Churg A: Animal models of chronic obstructive pulmonary disease. Am J Physiol Lung Cell Mol Physiol 295: L1-L15, 2008.

21. Feng J, Wang QS, Chiang A and Chen BY: The effects of sleep hypoxia on coagulant factors and hepatic inflammation in emphysematous rats. PLoS One 5: e13201, 2010.

22. Barreiro E, Peinado VI, Galdiz JB, et al: Cigarette smoke-induced oxidative stress: A role in chronic obstructive pulmonary disease skeletal muscle dysfunction. Am J Respir Crit Care Med 182: 477-488, 2010

23. Fukushima H, Matsumoto A, Inuzuka H, et al: SCF (Fbw7) modulates the NFkB signaling pathway by targeting NFkB2 for ubiquitination and destruction. Cell Rep 1: 434-443, 2012.

24. Wang T, Leng YF, Zhang Y, Xue X and Kang YQ: Oxidative stress and hypoxia-induced factor 1alpha expression in gastric ischemia. World J Gastroenterol 17: 1915-1922, 2011.

25. Engelhardt S, Al-Ahmad AJ, Gassmann M and Ogunshola OO Hypoxia selectively disrupts brain microvascular endothelial tight junction complexes through a hypoxia-inducible factor-1 (HIF-1) dependent mechanism. J Cell Physiol 229: 1096-1105, 2014.
26. Assimakopoulos SF, Tsamandas AC, Tsiaoussis GI, et al Altered intestinal tight junctions' expression in patients with liver cirrhosis: a pathogenetic mechanism of intestinal hyperpermeability. Eur J Clin Invest 42: 439-446, 2012.

27. Zhang YG, Wu S, Xia Y and Sun J: Salmonella infection upregulates the leaky protein claudin-2 in intestinal epithelial cells. PloS One 8: e58606, 2013.

28. Guo L, Li L, Wang W, Pan Z, Zhou Q and Wu Z: Mitochondrial reactive oxygen species mediates nicotine-induced hypoxia-inducible factor-1alpha expression in human non-small cell lung cancer cells. Biochim Biophys Acta 1822: 852-861, 2012.

29. Robinson A, Keely S, Karhausen J, Gerich ME, Furuta GT and Colgan SP: Mucosal protection by hypoxia-inducible factor prolyl hydroxylase inhibition. Gastroenterology 134: 145-155, 2008.

30. Feinman R, Deitch EA, Watkins AC, et al: HIF-1 mediates pathogenic inflammatory responses to intestinal ischemia-reperfusion injury. Am J Physiol Gastrointest Liver Physiol 299: G833-G843, 2010.

31. Koury J, Deitch EA, Homma H, et al: Persistent HIF-1alpha activation in gut ischemia/reperfusion injury: potential role of bacteria and lipopolysaccharide. Shock 22: 270-277, 2004.

32. Cao M, Wang P, Sun C, He W and Wang F: Amelioration of IFN-gamma and TNF-alpha-induced intestinal epithelial barrier dysfunction by berberine via suppression of MLCK-MLC phosphorylation signaling pathway. PLoS One 8: e61944, 2013.

33. McGilligan VE, Wallace JM, Heavey PM, Ridley DL and Rowland IR: The effect of nicotine in vitro on the integrity of tight junctions in Caco-2 cell monolayers. Food Chem Toxicol 45: 1593-1598, 2007

34. Wang H, Zhao JX, Hu N, Ren J, Du M and Zhu MJ: Side-stream smoking reduces intestinal inflammation and increases expression of tight junction proteins. World J Gastroenterol 18: 2180-2187, 2012 .

35. Verschuere S, Bracke KR, Demoor T, et al: Cigarette smoking alters epithelial apoptosis and immune composition in murine GALT. Lab Invest 91: 1056-1067, 2011.

36. Sowter HM, Ratcliffe PJ, Watson P, Greenberg AH and Harris AL: HIF-1-dependent regulation of hypoxic induction of the cell death factors BNIP3 and NIX in human tumors. Cancer Res 61: 6669-6673, 2001.

37. Boyd JM, Malstrom S, Subramanian T, et al: Adenovirus E1B $19 \mathrm{kDa}$ and $\mathrm{Bcl}-2$ proteins interact with a common set of cellular proteins. Cell 79: 341-351, 1994.

38. Mazzon E and Cuzzocrea S: Role of TNF-alpha in ileum tight junction alteration in mouse model of restraint stress. Am J Physiol Gastrointest Liver Physiol 294: G1268-G1280, 2008.

39. Guo X, Ko JK, Mei QB and Cho CH: Aggravating effect of cigarette smoke exposure on experimental colitis is associated with leukotriene $\mathrm{B}(4)$ and reactive oxygen metabolites. Digestion 63: 180-187, 2001.

40. Lee IT and Yang CM: Role of NADPH oxidase/ROS in pro-inflammatory mediators-induced airway and pulmonary diseases. Biochem Pharmacol 84: 581-590, 2012. 